\title{
Some Groupoids and their Representations by Means of Integer Sequences
}

\author{
Amelia Carolina Sparavigna ${ }^{1}$
}

\author{
${ }^{1}$ Department of Applied Science and Technology, Politecnico di Torino, Torino, Italy
}

\begin{abstract}
In some previous works, we have discussed the groupoids related to the integer sequences of Mersenne, Fermat, Cullen, Woodall and other numbers. These groupoids possess different binary operators. As we can easily see, other integer sequences can have the same binary operators, and therefore can be used to represent the related groupoids. Using the On-Line Encyclopedia of Integer Sequences (OEIS), we are able to identify the properties of these representations of groupoids. At the same time, we can also find integer sequences not given in OEIS and probably not yet studied.
\end{abstract}

Keywords: Groupoid Representations, Integer Sequences, Binary Operators, Generalized Sums, Generalized Entropies, Tsallis Entropy, Q-Calculus, Abelian Groups, Fermat Numbers, Mersenne Numbers, Triangular Numbers, Repunits, Oblong Numbers

\begin{abstract}
Introduction
A groupoid is an algebraic structure made by a set with a binary operator [1]. The only restriction on the operator is closure. This property means that, applying the binary operator to two elements of a given set $S$, we obtain a value which is itself a member of S. If this binary operation is associative and we have a neutral element and opposite elements into the set, the groupoid becomes a group.
\end{abstract}

Groupoids are interesting also for the study of integer numbers. As shown in some previous works [2-7], the integer sequences of Mersenne, Fermat, Cullen, Woodall and other numbers are groupoid possessing different binary operators. Here we show that other integer sequences can have the same binary operators, and therefore can be used to represent the related groupoids. That is, we can obtain different integer sequences by means of the recurrence relations generated by the considered binary operations.

In [7], we started the search for different representations for the groupoid of Triangular Numbers. Here we generalize this search, using the binary operators obtained in the previous analyses. In particular, we will see the representations linked to Mersenne, Fermat, Cullen, Woodall, Carol and Kynea, and Oblong numbers. The binary operators of these numbers have been already discussed in previous works. The results concerning the Triangular numbers are also reported.

Using the On-Line Encyclopedia of Integer Sequences (OEIS), we are able to identify the several representations of groupoids. At the same time, we can also find integer sequences not given in OEIS and probably not yet studied.

\section{Mersenne numbers}

We discussed the binary operator of the set of Mersenne numbers in $[8,9]$. The numbers are given $\operatorname{as}_{n}=2^{n}-1$. The binary operator is:

$$
M_{n+m}=M_{n} \oplus M_{m}=M_{n}+M_{m}+M_{n} M_{m}(1)
$$

As shown in [9], this binary operation is a specific case of the binary operator of q-integers, which can be linked to the generalized sum of Tsallis entropy $[10,11]$.

The binary operator can be used to have a recurrence relation:

$$
M_{n+1}=M_{n} \oplus M_{1}
$$

Here in the following, let us show the sequences that we can generate from (1) and (2).

We use OEIS, the On-Line Encyclopedia of Integer Sequences, to give more details on them.

$M_{1}=0$, sequence $0,0,0,0,0,0, \ldots$.

$M_{1}=1$, sequence $3,7,15,31,63,127,255,511$, $1023,2047,4095,8191,16383,32767,65535$, 131071, 262143, 524287, 1048575, 2097151, and so on. The Mersenne numbers $2^{n}-1$. This sequence is OEIS A000225. (OEIS tells that these numbers are sometimes called Mersenne numbers, "although that name is usually reserved for A001348").

This article is published under the terms of the Creative Commons Attribution License 4.0

Author(s) retain the copyright of this article. Publication rights with Alkhaer Publications.

Published at: http://www.ijsciences.com/pub/issue/2019-10/

DOI: 10.18483/ijSci.2188; Online ISSN: 2305-3925; Print ISSN: 2410-4477 
$M_{1}=2$, sequence $8,26,80,242,728,2186,6560$, 19682, 59048, 177146, 531440, 1594322, 4782968, $14348906,43046720,129140162,387420488$, and so on (OEIS A024023, $\left.a_{n}=3^{n}-1\right)$.

$M_{1}=3$, sequence $15,63,255,1023,4095,16383$, $65535,262143,1048575,4194303,16777215$, 67108863, 268435455, and so on (OEIS A046092, $\left.a_{n}=4^{n}-1\right)$.

And we can continue: $M_{1}=4$, OEIS A024049, $a_{n}=5^{n}-1 ; M_{1}=5$, OEIS A024062, $a_{n}=6^{n}-1$ ; $M_{1}=6$ OEIS A024075, $a_{n}=7^{n}-1$, and so on. An interesting sequence is $M_{1}=9, \mathrm{~A} 002283$, $a_{n}=10^{n}-1$. Dividing this sequence by 9 , we have the repunits A002275, $a_{n}=\left(10^{n}-1\right) / 9$. The generalized sum of the repunits is given in [12].

\section{Fermat numbers}

The group of Fermat numbers has been discussed in [13]. As explained in [14], there are two definitions of the Fermat numbers. "The less common is a number of the form $2^{n}+1$ obtained by setting $\mathrm{x}=1$ in a Fermat polynomial, the first few of which are 3, 5, 9, 17, 33, ... (OEIS A000051)" [14]. We used this definition.

$$
\begin{gathered}
F_{n}=2^{n}+1 \\
F_{n+m}=F_{n} \oplus F_{m}=\left(1-F_{n}\right)+\left(1-F_{m}\right)+F_{n} F_{m}(3)
\end{gathered}
$$

The binary operator can be used to have a recurrence relation: $F_{n+1}=F_{n} \oplus F_{1}$

Sequences can generate from (3) and (4).

$F_{1}=0$, sequence $2,0,2,0,2,0, \ldots$;

$F_{1}=1$, sequence $1,1,1,1,1,1, \ldots$.

$F_{1}=2$, sequence $2,2,2,2,2,2, \ldots$.

$F_{1}=3$, sequence $5,9,17,33,65,129,257,513$, $1025,2049,4097,8193,16385,32769,65537$, 131073, 262145, 524289, 1048577, 2097153, and so on, the Fermat numbers. (OEIS A000051, $a_{n}=2^{n}+$ 1.

$F_{1}=4$, sequence $\mathrm{A} 034472, a_{n}=3^{n}+1$; for $F_{1}=5$, sequence A052539, $a_{n}=4^{n}+1$. Continuing with 6 , we have A034474, $a_{n}=5^{n}+1$. For 7 , we have A062394, $a_{n}=6^{n}+1$. And so on.

\section{Cullen and Woodall numbers}

These numbers had been studied in [15].

Let us consider the Cullen numbers, $C_{n}=n 2^{n}+1$. We have the binary operator:

$$
\begin{gathered}
C_{n+m}=C_{n} \oplus C_{m}=\left(\frac{1}{n}+\frac{1}{m}\right)\left(C_{n}-1\right)\left(C_{m}-1\right)+1 \\
C_{n+1}=C_{n} \oplus C_{1}
\end{gathered}
$$

$C_{1}=1$, sequence $1,1,1,1,1,1,1$, and so on.

$C_{1}=2$, sequence $3,4,5,6,7,8,9$, and so on.

$C_{1}=3$, sequence $9,25,65,161,385,897,2049$, $4609,10241,22529,49153,106497,229377$, 491521, 1048577, 2228225, 4718593, 9961473 , 20971521, 44040193, and so on. OEIS A002064, Cullen numbers: $a_{n}=n 2^{n}+1$.

$C_{1}=4$, sequence $19,82,325,1216,4375,15310$, 52489, 177148, 590491, 1948618, 6377293, 20726200, 66961567, 215233606, 688747537, and so on. OEIS A050914, $a_{n}=n 3^{n}+1$. For $C_{1}=5$ sequence A050915, $a_{n}=n 4^{n}+1$. And so on. Let us mention the case $C_{1}=11$ which is giving A064748, $a_{n}=n 10^{n}+1$. That is: 201, 3001, 40001, 500001, 6000001, 70000001, 800000001, and so on.

Woodall numbers are $W_{n}=n 2^{n}-1$, and the binary operator is:

$$
\begin{gathered}
W_{n+m}=W_{n} \oplus W_{m}=\left(\frac{1}{n}+\frac{1}{m}\right)\left(W_{n}+1\right)\left(W_{m}+1\right)-1 \\
W_{n+1}=W_{n} \oplus W_{1}
\end{gathered}
$$

$W_{1}=0$, sequence $1,2,3,4,5,6,7$, and so on.

$W_{1}=1, \quad$ sequence $7,23,63,159,383,895,2047$, 4607, 10239, 22527, 49151, 106495, 229375, 491519, 1048575, 2228223, 4718591, 9961471, 20971519, 44040191, and so on. A003261, Woodall (or Riesel) numbers: $a_{n}=n 2^{n}-1$.

$W_{1}=2$, sequence A060352, $a_{n}=n 3^{n}-1$. For $W_{1}=3$, we have sequence A060416, $a_{n}=n 4^{n}-1$. And so on. Let us mention the case $W_{1}=9$, which is giving A064756, $a_{n}=n 10^{n}-1$, that is, 199, 2999, 39999, 499999, 5999999, 69999999, 799999999, and so on.

\section{Carol and Kynea Numbers}

These numbers have been studied in [3]. Carol number is: $C_{n}=\left(2^{n}-1\right)^{2}-2$. The binary operator $C_{n} \oplus C_{m}$ is given in [3]:

$$
C_{m} \oplus C_{n}=6+C_{m} C_{n}+3 C_{m}+3 C_{n}+a+b+c
$$

where $a=2\left(C_{m}+2\right)\left(C_{n}+2\right)^{1 / 2}, b=2\left(C_{m}+\right.$ $2)^{1 / 2}\left(C_{n}+2\right), c=2\left(C_{m}+2\right)^{1 / 2}\left(C_{n}+2\right)^{1 / 2}$.

We can use again $C_{n+1}=C_{n} \oplus C_{1}$. Since the binary operator contains square roots, we can obtain integer 
sequences only in some cases.

$C_{1}=-1$, sequence A093112, $a_{n}=\left(2^{n}-1\right)^{2}-2$, that is $7,47,223,959,3967,16127,65023,261119$, 1046527, 4190207, ... As told in [16], Cletus Emmanuel called these numbers as "Carol numbers".

$C_{1}=2$, sequence $62,674,6398,58562,529982$, 4778594, 43033598, 387381122, 3486666302, 31380705314 , and so on. Not given in OEIS.

$C_{1}=7$, sequence 223, 3967, 65023, 1046527, $16769023, \quad 268402687, \quad 4294836223$, 68718952447, 1099509530623, 17592177655807, and so on. Not given in OEIS.

Let us consider the Kynea numbers.

$$
K_{n}=\left(2^{n}+1\right)^{2}-2
$$

The binary operator $K_{n} \oplus K_{m}$ is given in [3]. We use again $K_{n+1}=K_{n} \oplus K_{1}$. Again, we have square roots, so we can obtain integer sequences only in some cases.

$K_{1}=-1$, sequence $-1,-1,-1,-1,-1$, and so on.

$K_{1}=2$, sequence $2,2,2,2,2,2$, and so on.

$K_{1}=7$, sequence A093069,$a_{n}=\left(2^{n}+1\right)^{2}-2$, that is $7,23,79,287,1087,4223,16639,66047$, 263167, 1050623, 4198399, and so on. As told in [17], Cletus Emmanuel calls these "Kynea numbers" [17].

$K_{1}=14$, sequence $98,782,6722,59534,532898$, 4787342, 43059842, 387459854, 3486902498, 31381413902 , and so on. Not given in OEIS.

\section{Oblong numbers}

These numbers are discussed in [4]. The oblong number is defined as: $O_{n}=n(n+1)$. It is given by OEIS A002378. An oblong number is also known as a promic, pronic, or heteromecic number. OEIS gives the list: $2,6,12,20,30,42,56,72,90,110,132,156$, $182,210,240,272,306,342,380,420,462,506$, $552,600,650,702,756,812,870,930,992,1056$, and so on.

The binary operator $O_{n} \oplus O_{m}$ is:

$$
O_{m} \oplus O_{n}=\frac{1}{2}+O_{m}+O_{n}+a+b
$$

where $a=2\left(O_{m}+1 / 4\right)^{1 / 2}\left(O_{n}+1 / 4\right)^{1 / 2}, b=$ $-\left(O_{m}+1 / 4\right)^{1 / 2}-\left(O_{n}+1 / 4\right)^{2}$. Again, as we did before we have:
$O_{1}=0$, sequence $0,0,0,0,0$, and so on

$O_{1}=2$, sequence OEIS A002378, as given above.

$O_{1}=6$, sequence, A002943, $a_{n}=2 n(2 n+1)$.

$O_{1}=12$, sequence A045945, Hexagonal matchstick numbers: $a_{n}=3 n(3 n+1)$.

$O_{1}=20$, sequence $72,156,272,420,600,812$, $1056,1332,1640,1980$, and so on. Not given in OEIS. It is $a_{n}=4 n(4 n+1)$. And we can continue.

Of course, we can repeat the same approach for the odd squares (A016754) numbers. Their binary operator is given in [4]. Also for the centered square numbers and the star numbers, we have the binary operators [5,6], so we can find the related representations by means of integer sequences too. As previously told, among the generated sequences, news sequences are produced that can be interesting for further investigation of integer sequences.

\section{Triangular numbers}

These numbers are really interesting. The numbers are of the form (OEIS A000217):

$$
T_{n}=\sum_{k=1}^{n} k=\frac{n(n+1)}{2}
$$

I have discussed them in [7]. For these numbers we can give two binary operators. For the convenience of the reader, I show the results that we can obtain.

The first binary operator is [7]:

$$
\begin{aligned}
& T_{n} \oplus T_{m}=T_{n}+T_{m}+\frac{1}{4}\left[1-\left(1+8 T_{n}\right)^{1 / 2}-\right. \\
& \left.\left(1+8 T_{m}\right)^{1 / 2}+\left(1+8 T_{n}\right)^{1 / 2}\left(1+8 T_{m}\right)^{1 / 2}\right]
\end{aligned}
$$

Again we consider $T_{n+1}=T_{n} \oplus T_{1}$, and change the value of $T_{1}$. Here in the following the sequences that we generate.

$T_{1}=0$, sequence $0,0,0,0,0,0, \ldots$.

$T_{1}=1$, sequence $3,6,10,15,21,28,36,45,55$, $66,78,91,105,120,136,153,171,190,210$, 231, and so on. And this is OEIS A000217, the sequence of triangular numbers.

$T_{1}=3$, sequence $10,21,36,55,78,105,136,171$, $210,253,300,351,406,465,528,595,666,741$, $820,903, \ldots$. Searching this sequence in OEIS, we can easily find that it is A014105, that is, the Second Hexagonal Numbers: $H_{n}=n(2 n+1)$.

$T_{1}=4$, sequence $12,24,40,60,84,112,144,180$ 
$220,264,312,364,420,480,544,612,684,760$, $840,924, \ldots$ OEIS A046092 (four times triangular numbers).

$T_{1}=6$, sequence $21,45,78,120,171,231,300,378$, 465, 561, 666, 780, 903, 1035, 1176, 1326, 1485 , 1653, 1830, 2016, ... OEIS A081266 (Staggered diagonal of triangular spiral in A051682).

$T_{1}=7$, sequence $23,48,82,125,177,238,308,387$, $475,572,678,793,917,1050,1192,1343,1503$, $1672,1850,2037, \ldots$ OEIS A062725.

$T_{1}=10$, sequence $36,78,136,210,300,406,528$,
$666,820,990,1176,1378,1596,1830,2080,2346$ $2628,2926,3240,3570, \ldots$ OEIS A033585, that is, numbers: $2 n(4 n+1)$.

$T_{1}=11$, sequence $38,81,140,215,306,413,536$, 675, 830, 1001, 1188, 1391, 1610, 1845, 2096, 2363 , $2646,2945,3260,3591, \ldots$ OEIS A139276, that is, numbers $n(8 n+3)$.

Of course, we can continue and obtain further sequences.

As previously told, we have a second binary operator for the triangular numbers [7]. It is the following:

$$
T_{n} \oplus T_{m}=T_{n}+T_{m}+\frac{1}{4}\left[1+\left(1+8 T_{n}\right)^{1 / 2}+\left(1+8 T_{m}\right)^{1 / 2}+\left(1+8 T_{n}\right)^{1 / 2}\left(1+8 T_{m}\right)^{1 / 2}\right]
$$

Again, let us consider $T_{n+1}=T_{n} \oplus T_{1}$ as we did before.

$T_{1}=0$, sequence $1,3,6,10,15,21,28,36,45$, $55,66,78,91,105,120,136,153,171,190,210$, and so on. OEIS A000217, the sequence of triangular numbers.

$T_{1}=1$, sequence $6,15,28,45,66,91,120,153$, 190, 231, 276, 325, 378, 435, 496, 561, 630, 703, $780,861, \ldots$ OEIS A000384, Hexagonal numbers $H_{n}=n(2 n-1)$.

$T_{1}=3$, sequence $15,36,66,105,153,210,276$, $351,435,528,630,741,861,990,1128,1275,1431$, $1596,1770,1953, \ldots$ OEIS A062741, three times pentagonal numbers $3 n(3 n-1) / 2$.

$T_{1}=4$, sequence $17,39,70,110,159,217,284$, 360, 445, 539, 642, 754, 875, 1005, 1144, 1292, $1449,1615,1790,1974, \ldots$ OEIS A022266, numbers $n(9 n-1) / 2$.

$T_{1}=6$, sequence $28,66,120,190,276,378,496$, 630, 780, 946, 1128, 1326, 1540, 1770, 2016, 2278, $2556,2850,3160,3486, \quad \ldots \quad$ OEIS A014635, numbers $2 n(4 n-1)$.

$T_{1}=7$, sequence $30,69,124,195,282,385,504$, 639, 790, 957, 1140, 1339, 1554, 1785, 2032, 2295, 2574, 2869, 3180, 3507, ... OEIS A139274, numbers $n(8 n-1)$.

$T_{1}=10$, sequence $45,105,190,300,435,595,780$, $990,1225,1485,1770,2080,2415,2775,3160$, $3570,4005,4465,4950,5460 \ldots$ This sequence is not present in OEIS.
$T_{1}=11$, sequence $47,108,194,305,441,602,788$, $999,1235,1496,1782,2093,2429,2790,3176$, 3587, 4023, 4484, 4970, 5481, .... OEIS A178572, numbers with ordered partitions that have periods of length 5 .

Using the On-Line Encyclopedia of Integer Sequences (OEIS), we have seen that quite different sequences can have the same binary operators. We have also found integer sequences not given in OEIS and that need to be studied.

\section{Conclusion}

Groupoids are related to the integer sequences. These groupoid possess different binary operators. As we have shown, other integer sequences can have the same binary operators, and therefore can be used to represent the related groupoids.

\section{References}

1. Stover, Christopher and Weisstein, Eric W. "Groupoid." From MathWorld--A Wolfram Web Resource. http://mathworld.wolfram.com/Groupoid.html

2. Sparavigna, A. C. (2019). Composition Operations of Generalized Entropies Applied to the Study of Numbers, International Journal of Sciences, Vol. 8, Issue 4, pp. 87-92. DOI: $10.18483 / \mathrm{ijSci} .2044$

3. Sparavigna, A. C. (2019). Binary Operators of the Groupoids of OEIS A093112 and A093069 Numbers (Carol and Kynea Numbers). Zenodo. June 6. DOI: 10.5281/zenodo.3240465

4. Sparavigna, A. C. (2019). Groupoids of OEIS A002378 and A016754 Numbers (oblong and odd square numbers). Zenodo. June 16. DOI: 10.5281 /zenodo. 3247003

5. Sparavigna, A. C. (2019. Groupoid of OEIS A001844 Numbers (centered square numbers). Zenodo. June 22. DOI: 10.5281/zenodo.3252339

6. Sparavigna, A. C. (2019). Groupoid of OEIS A003154 Numbers (star numbers or centered dodecagonal numbers). Zenodo. Septembr 5. DOI: 10.5281/zenodo.3387054

7. Sparavigna, A. C. (2019,. The groupoid of the Triangular Numbers and the generation of related integer sequences. Zenodo, October 2. DOI: 10.5281/zenodo.3470205 
8. Sparavigna, A. C. (2018, May 20). On a generalized sum of the Mersenne Numbers. Zenodo, May 20. DOI: 10.5281/zenodo. 1250048

9. Sparavigna, A. C. (2018). The q-Integers and the Mersenne Numbers. SSRN Electronic Journal, May 23. DOI: $10.2139 / \mathrm{ssrn} .3183800$

10. Tsallis, C. (1988). Possible Generalization of BoltzmannGibbs Statistics, Journal of Statistical Physics, Vol. 52, pp. 479-487. DOI:10.1007/BF01016429

11. Curado, E. M., Tempesta, P., \& Tsallis, C. (2016). A new entropy based on a group-theoretical structure. Annals of Physics, Vol. 366, pp. 22-31. DOI: 10.1016/j.aop.2015.12.008
12. Sparavigna, A. C. (2019). On Repunits. Zenodo, April 14. DOI: 10.5281/zenodo.2639620

13. Sparavigna, A. C. (2018). The group of the Fermat Numbers Zenodo, May 24. DOI: 10.5281/zenodo. 1252422

14. Weisstein, Eric W. "Fermat Number." From MathWorld--A Wolfram Web Resource. http://mathworld.wolfram.com/FermatNumber.html

15. Sparavigna, A. C. (2019). On the generalized sums of Mersenne, Fermat, Cullen and Woodall Numbers. Zenodo, April 9. DOI: 10.5281/zenodo.2634312

16. https://oeis.org/A093112

17. https://oeis.org/A093069 